\title{
Association of sleep problems with neuroendocrine hormones and coagulation factors in patients with acute myocardial infarction
}

Roland von Känel ${ }^{1^{*}}$ (D), Mary Princip ${ }^{1}$, Jean-Paul Schmid² ${ }^{2}$ Jürgen Barth ${ }^{3}$, Hansjörg Znoj ${ }^{4}$, Ulrich Schnyder ${ }^{5}$ and Rebecca E. Meister-Langraf ${ }^{6}$

\begin{abstract}
Background: Obstructive sleep apnea (OSA) and insomnia are frequent sleep problems that are associated with poor prognosis in patients with coronary heart disease. The mechanisms linking poor sleep with an increased cardiovascular risk are incompletely understood. We examined whether a high risk of OSA as well as insomnia symptoms are associated with neuroendocrine hormones and coagulation factors in patients admitted with acute myocardial infarction.

Methods: We assessed 190 patients (mean age 60 years, 83\% men) in terms of OSA risk (STOP screening tool for the assessment of high vs. low OSA risk) and severity of insomnia symptoms (Jenkins Sleep Scale for the assessment of subjective sleep difficulties) within $48 \mathrm{~h}$ of an acute coronary intervention. Circulating concentrations of epinephrine, norepinephrine, cortisol, fibrinogen, D-dimer, and von Willebrand factor were measured the next morning. The association of OSA risk and insomnia symptoms with neuroendocrine hormones and coagulation factors was computed using multivariate models adjusting for demographic factors, health behaviors, somatic and psychiatric comorbidities, cardiac disease-related variables, and OSA risk in the model for insomnia symptoms, respectively, for insomnia symptoms in the model for OSA risk.
\end{abstract}

Results: High OSA risk was identified in $41 \%$ of patients and clinically relevant insomnia symptoms were reported by $27 \%$ of patients. Compared to those with low OSA risk, patients with high OSA risk had lower levels of epinephrine $(p=0.015)$, norepinephrine $(p=0.049)$ and cortisol $(p=0.001)$. More severe insomnia symptoms were associated with higher levels of fibrinogen $(p=0.037)$, driven by difficulties initiating sleep, and with lower levels of norepinephrine $(p=0.024)$, driven by difficulties maintaining sleep.

Conclusions: In patients with acute myocardial infarction, sleep problems are associated with neuroendocrine hormones and coagulation activity. The pattern of these relationships is not uniform for patients with a high risk of OSA and those with insomnia symptoms, and whether they contribute to adverse cardiovascular outcomes needs to be established.

Trial registration: ClinicalTrials.gov NCT01781247.

Keywords: Acute coronary syndrome, Biomarker , Blood coagulation , HPA axis, Insomnia, Sleep apnea, Sympathetic nervous system

\footnotetext{
* Correspondence: roland.vonkaenel@usz.ch

${ }^{1}$ Department of Consultation-Liaison Psychiatry and Psychosomatic Medicine,

University Hospital Zurich, Culmannstrasse 8, CH-8091 Zurich, Switzerland

Full list of author information is available at the end of the article
}

(c) The Author(s). 2018 Open Access This article is distributed under the terms of the Creative Commons Attribution 4.0 International License (http://creativecommons.org/licenses/by/4.0/) which permits unrestricted use, distribution, and reproduction in any medium, provided you give appropriate credit to the original author(s) and the source, provide a link to the Creative Commons license, and indicate if changes were made. The Creative Commons Public Domain Dedication waiver (http://creativecommons.org/publicdomain/zero/1.0/) applies to the data made available in this article, unless otherwise stated. 


\section{Background}

Obstructive sleep apnea (OSA) is a common sleep-related breathing disorder that is caused by repeated upper airway obstruction during sleep and characterized through symptoms of snoring, breathing cessations while sleeping, and daytime sleepiness and fatigue [1]. Insomnia disorders are characterized by subjective sleep initiating and maintaining problems in spite of adequate circumstances to sleep and daytime impairment, whereas insomnia symptoms alone do not merit a formal diagnosis of insomnia [2].

Obstructive sleep apnea and insomnia symptoms, including difficulty initiating sleep, difficulty maintaining sleep and non-restorative sleep, have been associated with an increased risk of incident coronary heart disease (CHD) events, independently of a range of other risk factors [3-7]. Sleep problems may also be associated with poor prognosis of CHD. In two meta-analyses, CHD patients with OSA had an almost two-fold higher risk of poor cardiovascular prognosis, including recurrent $\mathrm{CHD}$ events and stroke, than $\mathrm{CHD}$ patients without OSA $[8,9]$. In terms of insomnia symptoms in patients with acute myocardial infarction (MI), not feeling well-rested was predictive of case fatality in the 4 weeks post-MI in men and of 10-year risk of new CVD events in women [10]. In women, assessed 3-6 months after acute coronary syndrome (ACS), a sleep quality index, defined by insomnia symptoms was a predictor of recurrent CHD events at 5-year follow-up, after controlling for CVD risk factors and depressive symptoms [11]. In men who had undergone percutaneous coronary angioplasty, a state of vital exhaustion, characterized by subjective sleep disturbances, including troubles falling asleep, waking up repeatedly during the night, and not feeling well rested, along with profound feelings of fatigue, was predictive of new CHD events after a follow-up of 1.5 years [12].

Different biological mechanisms, initiated by intermittent hypoxia, are thought to underpin the association of OSA with an increased risk of incident CHD, including sympathetic hyperactivity, systemic inflammation, oxidative stress, vascular endothelial dysfunction, and hypercoagulability [13, 14]. Likewise, sympathetic hyperactivity and systemic inflammation, but also elevated cortisol (COR) levels have been proposed to be biological mechanisms potentially linking insomnia with incident CVD [15]. Although these mechanisms might also link poor sleep with recurrent atherothrombotic events in patents with established CHD, this has not systematically been explored. In the present study, we focused on circulating neuroendocrine and coagulation markers in patients with sleep problems admitted with acute MI, as sleep studies in individuals without CVD suggest a role of neuroendocrine changes and coagulation activation in incident atherothrombotic events. For instance, in middle-aged and elderly subjects, objective sleep problems, including greater apnea-hypopnea index and oxygen desaturation, indicating OSA, as well as poor subjective sleep quality, were associated with increased plasma levels of fibrinogen, D-dimer, and von Willebrand factor (VWF) [16-20]. In patients with ACS, these prothrombotic markers were of prognostic value for major cardiovascular events and all-cause mortality [21-24].

In OSA, hyperactivity of the sympathetic nervous system (SNS) is evidenced by increased norepinephrine (NEPI) turnover [25], whereas findings on hypothalamic-pituitary adrenal (HPA) axis function, including COR levels, are contradictory [26]. In insomnia, HPA axis dysregulation is evidenced by high COR levels in the evening, affecting sleep architecture [27], and low morning COR levels, which relate to poor sleep quality, nightly awakenings and unrefreshing sleep [28]. The metabolic consequences of neuroendocrine dysregulation include hypertension, dyslipidemia, obesity, diabetes and a prothrombotic state, which are associated with the progression of atherothrombotic disease [29]. Secretion of epinephrine (EPI), NEPI, and COR has effects on coagulation, including increases in fibrinogen, D-dimer and VWF levels [30, 31], and neuroendocrine dysregulation might partially account for a link between sleep problems and coagulation activity [17].

Insomnia and OSA are often found together in the same patient, with up to $50 \%$ of OSA patients having insomnia symptoms; a similar prevalence of at least mild OSA is found in patients with insomnia [32]. However, in meta-analyses of prospective studies incident and recurrent CVD risk were not adjusted for the other sleep disorder nor were joint effects investigated [3-9]. As yet, except for hypertension [33], there is little evidence that OSA and insomnia together are associated with a greater risk of CVD or frequency of CVD risk factors than OSA alone [34]. However, such studies are still few in number and, to our knowledge, researchers did not investigate neuroendocrine and coagulation activity.

In this study we aimed to examine the association of a high risk of OSA and insomnia symptoms, independently of each other, with neuroendocrine hormones and coagulation factors in patients with acute MI. We specifically hypothesized that more sleep problems would be associated with higher levels of fibrinogen, D-dimer and VWF. We specified no a priori hypothesis regarding the direction of an association of sleep problems with EPI, NEPI and COR levels; this is because findings vary across studies, depending upon circadian factors, natural vs. experimental designs, confounding variables, and measures of sleep quantity and quality [26, 35, 36]. We predicted the hypothesized relationships to be independent of demographics, comorbidity, health behaviors, and cardiac-related variables. In complementary analyses we examined whether neuroendocrine hormones may account 
for the relation between sleep problems and coagulation factors, and whether there were joint effects of OSA risk and insomnia symptoms for neuroendocrine and coagulation outcomes.

\section{Methods \\ Study participants and design \\ Rationale of the parent study}

Data for this ancillary sleep study were collected from patients who participated in the Myocardial Infarction-Stress Prevention Intervention (MI-SPRINT) randomized controlled trial [37]. The primary aim of the parent study was to test whether a psychological first aid approach (i.e., one single 45-min session of psychological counseling at hospital referral) may prevent the incidence of ACS-induced posttraumatic stress at 3-month follow-up [37]. About $12 \%$ of ACS patients develop clinically significant levels of posttraumatic stress which is a predictor of poor cardiac prognosis [38]. Disrupted sleep could be one mechanism to explain this link [39]. The overarching hypothesis was that patients undergoing trauma-focused counseling would show better mental and physical health, including sleep, at follow-up than patients undergoing general stress counseling [37]. The intervention was not considered for the present study, as it showed no significant association with sleep, neuroendocrine and coagulation outcomes at hospital admission.

\section{Patient recruitment}

In brief, for MI-SPRINT, we recruited 190 patients with verified acute ST-elevation MI (STEMI) or non-STEMI and referred for acute coronary care intervention to the Bern University Hospital ("Inselspital") between January 2013 and September 2015. The diagnostic criteria and therapy for acute STEMI and non-STEMI followed the European Society of Cardiology guidelines on myocardial revascularization, including indications for primary percutaneous coronary intervention in non-STEMI, antithrombotic treatments, and treatments to establish stable hemodynamic conditions $[40,41]$. Inclusion criteria were 18 years or older, stable hemodynamic conditions, and a high level of acute distress during MI defined by scores of at least 5 for chest pain plus at least 5 for fear of dying and/or helplessness on numeric rating scales from 0 to 10 [37]. Exclusion criteria were emergency coronary artery bypass grafting, comorbid diseases likely to cause death within 1 year, cognitive impairment, severe clinical depression, suicidal ideations in the prior 2 weeks, inadequate knowledge of German, or participation in another trial. The study protocol was approved by the ethics committee of the State of Bern (KEK-Nr. 170/12). All patients were informed and gave signed consent.

\section{Data collection}

Within $48 \mathrm{~h}$ after having reached stable hemodynamic conditions, all included patients underwent a structured clinical interview on the coronary care unit on which occasion a medical history, sleep-related data and information on health behaviors were collected. To limit patient burden in this acute clinical setting, we decided to assess sleep problems with just two short screening tools, one to rate OSA risk, and one to rate the severity of sleep difficulties in terms of insomnia symptoms (see below). Cardiac-related variables were additionally abstracted from hospital charts. Fasting venous blood samples were collected for the measurement of neuroendocrine hormones and coagulation factors the next morning at $6 \mathrm{am}$. For logistical reasons and reasons of patient care, blood was collected non-fasting in 12 cases and not at $6 \mathrm{am}$ in 46 cases. Specific collection times were between 1 am and $5 \mathrm{am}(n=14)$, at $6 \mathrm{am}(n=144)$, between 7 am and $8 \mathrm{am}(n=13)$, between $9 \mathrm{am}$ and $1 \mathrm{pm}(n=$ $10)$, and between $2 \mathrm{pm}$ and $6 \mathrm{pm}(n=9)$. Collection time (all $p$-values >0.64) and fasting state (all p-values $>0.05)$ were not significantly associated with circulating levels of any neuroendocrine hormone or coagulation factor.

\section{Measures}

\section{Sleep problems}

To rate the risk of OSA, we applied a slightly modified version of the STOP questionnaire that is a tool to screen patients for OSA, asking for snoring (S); tiredness $(\mathrm{T})$, observed $(\mathrm{O})$ breathing cessations during sleep; and high blood pressure (P) [42]. The STOP questionnaire has been validated in patients with CHD [43] and in German [44]. The STOP questionnaire is particularly sensitive to identify patients with more severe forms of OSA [45], including in CHD patients [43]. Specific questions in our study (slight modifications from the original tool) asked about a history of snoring (irrespective of its loudness); history of breathing cessations while sleeping (although not necessarily observed); being often fatigued after sleep or while awake (sparing sleepiness); and history of hypertension (sparing treatment). Patients answering "yes" to at least two of these four questions were classified with a "high OSA risk", and all others with a "low OSA risk" [42]. To verify the accuracy of the STOP screening tool to a certain extent, we also inquired about a positive history of sleep apnea, predicting that patients who confirmed would largely fall into the group with a high OSA risk. We also asked patients whether they lived with someone to account for the fact that household members often notice and communicate signs of sleep-disordered 
breathing. Patients identified with a high OSA risk were not treated for a presumed sleep disorder during their acute hospitalization.

Sleep difficulties in terms of insomnia symptoms in the previous 4 weeks were inquired with the 4-item Jenkins Sleep Scale with questions about difficulties initiating sleep, frequent awakenings during the night, difficulties maintaining sleep, and non-restorative sleep (i.e., feeling tired and worn out in the morning after a usual night's sleep) [46]. Response alternatives (scores) are: not at all (0), 1-3 days (1), 4-7 days (2), 8-14 days (3), 15-21 days (4), and 22-31 days (5), yielding an average total score of all sleep difficulties between 0 and 5. Lower scores are indicative of better sleep quality. For illustrative purposes, patients were categorized in those with poor (sore $>2$ ) vs. good (score $\leq 2$ ) sleep. Cronbach's alpha was 0.70 in the present study, suggesting acceptable internal consistency of the scale.

\section{Neuroendocrine hormones}

Serum COR (nmol/L) was determined with an electro-chemiluminescence immunoassay on a Cobas analyzer (Roche Diagnostics, Switzerland) at the Institute of Clinical Chemistry, Inselspital, Bern University Hospital, Switzerland. EPI and NEPI concentrations were quantified in EDTA plasma by high-pressure liquid chromatography using electrochemical detection [47] at the Laboratory for Stress Monitoring, Göttingen, Germany (inter-/intra-assay coefficients of variation $<10 \%$; limit of detection $10 \mathrm{pg} / \mathrm{mL}$ ). Undetectable EPI levels $(n=16)$ were assigned half the limit of detection $(5 \mathrm{pg} / \mathrm{mL})$.

\section{Coagulation factors}

Following a strict in-house protocol to ensure adequate preanalytical conditions, all coagulation factors were determined in citrate plasma at the haemostasis laboratory, Inselspital, Bern University Hospital, Switzerland. Fibrinogen measurements $(\mathrm{g} / \mathrm{L})$ were performed according to the Clauss method (Dade ${ }^{\circ}$ Thrombin, Dade Behring, Liederbach, Germany). Plasma D-dimer levels (ng/mL) were determined with a quantitative sandwich enzyme immunoassay (VIDAS-D-Dimer, bioMérieux, Geneva, Switzerland), and from June 2015 on, with a particle-based immunoturbidimetric assay (Innovance ${ }^{\circ}$ D-Dimer; Siemens AG, Munich, Germany). A highly significant correlation (Passing Bablock regression analysis: $r=0.952$ ) between $\mathrm{D}$-dimer values assessed with these two methods has previously been demonstrated, suggesting that their analytical performance is equally good [48]. Von Willebrand factor antigen concentration (IU/ $\mathrm{dL}$ ) was determined with $\mathrm{vWF} \mathrm{Ag}^{\oplus}$ Kit (Siemens AG, Munich, Germany), an immunoturbidimetric method using polystyrene-based antibodies against VWF.

\section{Covariates}

In order to avoid overfitting of multivariate models, we included a selection of 16 potentially relevant predictors that might be confounders of the relationship between sleep problems and neuroendocrine measures and coagulation factors. We selected these covariates a priori based on the literature and on theoretical assumptions.

\section{Demographic factors}

Age and gender were abstracted from hospital charts. Socioeconomic status was based on the highest education level (high: high school graduation/matura, university graduation, including applied sciences; medium: apprenticeship or vocational school; low: lower than apprenticeship or vocational school) [49].

\section{Comorbidities}

We calculated the Charlson comorbidity index that provides an estimate of future mortality across several diseases [50]. Information on high cholesterol, hypertension, diabetes either with or without end organ damage, and lifetime depression was obtained through history taking.

\section{Health behaviors}

Patients disclosed their weight and height for the calculation of the body mass index. Smoking status was assessed in terms of current, former and never smokers, and physical activity ("that makes you sweat") in terms of the average frequency per week. Regarding alcohol consumption, we categorized patients into abstainers, moderate drinkers, and heavy drinkers ( $>21$ drinks/week for men, $>14$ drinks/week for women).

\section{Cardiac-related variables}

These included the type of MI (STEMI/non-STEMI), previous MI (yes/no), and the number of coronary arteries with stenosis $\geq 50 \%$. As an objective marker of MI severity, we calculated the Global Registry of Acute Coronary Events (GRACE) risk score from eight variables obtained at hospital admission: age, heart rate, systolic blood pressure, creatinine, Killip class, cardiac arrest, ST-segment deviation, and elevated cardiac enzymes [51]. The GRACE score is a robust predictor of the cumulative risk of death or death and recurrent MI from admission to 6 months after discharge [51].

\section{Acute distress}

The intensity of perceived distress during MI was assessed with three numeric rating scales (scores 0-10) for "pain intensity (during MI)", "fear of dying (until admission to the coronary care unit)" and "making sorrows and feeling helpless (when being told about having MI)" [37]. These measures were previously shown to 
predict CVD-related hospital readmissions in ACS patients after a 3-year follow-up [52]. The added sum score of the three numeric rating scales was divided by three to yield a severity index of acute distress.

\section{Data analysis}

Data were analyzed using SPSS 23.0 for Windows (SPSS Inc., Chicago, IL) with level of significance at $p<0.05$. For technical and logistic reasons (e.g., early discharge of patients), EPI and NEPI were missing in 45 patients, COR in 16 patients, fibrinogen in 14 patients, and D-dimer and VWF in 15 patients each. Due to lacking information, the GRACE risk score could not be computed for 18 patients. Seven or less values were missing for all other measures. We replaced all missing values with the expectation maximization algorithm to make use of all the available information from the total sample of 190 study participants. Supporting the adequacy of this approach, the strength and significance of the associations between OSA risk and COR $(r=-0.16, p=0.043 ; n=170)$ and between sleep difficulties and both fibrinogen $(r=$ $0.16, p=0.032 ; n=172)$ and NEPI $(r=-0.18, p=0.036 ; n$ $=141)$ in the patients with complete data for these measures were similar to those shown in the result section for the whole sample. Values of neuroendocrine hormones and coagulation factors were log-transformed before analysis.

Pearson correlation analysis was used to estimate the univariate relationship between two variables. Multivariate regression analysis was employed to identify whether OSA risk (categorical variable: high vs. low) and sleep difficulties (continuously scaled variable) were independently associated with neuroendocrine hormones and coagulation factors, after controlling for demographics, comorbidity, health behaviors, and cardiac-related variables, all entered in one block. If the total sleep difficultly scale showed a significant association, we computed post hoc analyses on each individual item. Finally, to test for a possible joint effect of OSA risk and sleep difficulties on outcomes, we additionally entered the interaction between the STOP score and the sleep difficulties score total into the regression equation. We allowed a maximum of 19 independent predicting variables to protect against model overfitting. Cook's distance and variance inflation factor, respectively, were used to verify the absence of influential outliers in the set of predictor variables and critical multicollinearity between two predictor variables, respectively.

We did not adjust $p$-values for multiple comparisons because of the pre-established primary hypothesis of a significant association of sleep problems with neuroendocrine and coagulation measures, and because of the risk of deeming truly important differences non-significant in a still nascent field of research [53]. However, partial correlation coefficients $\left(r_{p}\right)$ from the regression output were used to interpret effect sizes as small (0.1), medium (0.3), or large (0.5), irrespective of p-values [54].

\section{Results}

\section{Patient characteristics}

Table 1 shows the characteristics of the 190 patients, all of Caucasian ethnicity. The majority of participants were well-educated men with a first-time STEMI and living together with someone. The Charlson index indicated low comorbidity; nevertheless, a history of hypertension and high cholesterol were reported by every other patient, and a history of depression by almost 30\%. According to the GRACE score, the median (inter-quartile range) risk of 6-month death was 5\% (3-9). Regarding health behaviors, patients were on average overweight, and a substantial portion were current smokers and physically inactive. Defining the group of poor sleepers, a total of $52(27.4 \%)$ patients reported sleep difficulties on more than 7 days in the previous 4 weeks. A total of $77(40.5 \%)$ patients could be identified with a high OSA risk; 15 $(78.9 \%)$ of the 19 patients with a history of sleep apnea were in this group. Living with someone was not associated with OSA risk $(p=0.98)$, so it was not used as a covariate. The type of drugs delivered at admission and glucocorticoid use did not significantly differ between patients with high versus low OSA risk (all $p$-values $>0.06$ ).

\section{Unadjusted associations among sleep, neuroendocrine and coagulation measures}

There were several significant bivariate relationships between sleep problems, neuroendocrine and coagulation measures. High OSA risk was associated with more sleep difficulties $(r=0.20, p=0.006)$, with frequent nighttime awakenings $(r=0.30, p<0.001)$ and nonrestorative sleep $(r=0.19, p=0.011)$ driving this relationship. High OSA risk was also associated with lower COR levels $(r=-0.17, p=0.022)$, and more sleep difficulties were associated with higher fibrinogen levels $(r=0.14, p=0.048)$, with difficulties initiating sleep driving this relationship $(r=0.22, p=0.003)$.

Further expected direct associations were seen among all stress hormones (EPI and NEPI: $r=0.51$; EPI and COR: $\mathrm{r}=0.30$; NEPI and COR: 0.29; $p$-values < 0.001 ) and among all coagulation factors (fibrinogen and D-dimer: $r=0.35 ;$ fibrinogen and VWF; $r=0.38$; D-dimer and VWF: $\mathrm{r}=0.38$; $\mathrm{p}$-values $<0.001)$. Direct associations also emerged for NEPI with D-dimer $(r=0.17$, $p=0.020)$ and VWF $(r=0.16, p=0.033)$ levels, and between COR and fibrinogen levels $(r=0.21, p=0.004)$. 
Table 1 Demographic and clinical characteristics of the 190 study participants

\begin{tabular}{|c|c|}
\hline Age, yrs., M (SD) & $59.9(11.2)$ \\
\hline Sex (men), $n(\%)$ & $157(82.6)$ \\
\hline \multicolumn{2}{|l|}{ Education level } \\
\hline High, $n(\%)$ & $36(18.9)$ \\
\hline Medium, n (\%) & $136(71.6)$ \\
\hline Low, $n(\%)$ & $18(9.5)$ \\
\hline Living with someone, $n(\%)$ & $138(72.6)$ \\
\hline Charlson comorbidity index, M (SD) & $1.80(1.20)$ \\
\hline Positive history of hypertension, $n(\%)$ & $98(51.6)$ \\
\hline Positive history of high cholesterol, $n$ (\%) & $86(45.3)$ \\
\hline Positive history of depression, $n(\%)$ & $54(28.4)$ \\
\hline Body mass index, kg/m², M (SD) & $27.7(4.6)$ \\
\hline \multicolumn{2}{|l|}{ Smoking status } \\
\hline Current smoker, $n(\%)$ & $83(43.7)$ \\
\hline Former smoker, $n(\%)$ & $50(26.3)$ \\
\hline Never smoker, $n(\%)$ & $57(30.0)$ \\
\hline \multicolumn{2}{|l|}{ Alcohol consumption (drinks per week) } \\
\hline Abstainers, $n(\%)$ & $33(17.4)$ \\
\hline Moderate drinkers, $n$ (\%) & $147(77.4)$ \\
\hline Heavy drinkers, $n(\%)$ & $10(5.3)$ \\
\hline \multicolumn{2}{|l|}{ Physical activity (number of times per week) } \\
\hline $3-7, n(\%)$ & $49(25.8)$ \\
\hline $1-2, n(\%)$ & $52(27.4)$ \\
\hline$<1, n(\%)$ & $89(46.8)$ \\
\hline Previous myocardial infarction, $n$ (\%) & $20(10.5)$ \\
\hline Previous percutaneous coronary intervention & $27(14.2)$ \\
\hline Previous coronary artery bypass surgery & $5(2.6)$ \\
\hline \multicolumn{2}{|l|}{ Type of confirmed acute infarction } \\
\hline ST-elevation myocardial infarction, $n$ (\%) & $136(71.6)$ \\
\hline Non-ST-elevation myocardial infarction, $n$ (\%) & $54(28.4)$ \\
\hline $\begin{array}{l}\text { Major coronary arteries with stenosis } \geq 50 \% \\
\text { (number), M (SD) }\end{array}$ & $1.93(0.86)$ \\
\hline $\begin{array}{l}\text { Left ventricular ejection fraction } \\
\text { (angiography), \%, M (SD) }\end{array}$ & 47. $6(11.8)$ \\
\hline \multicolumn{2}{|l|}{ Vasopressant drugs at admission } \\
\hline Epinephrine, $n(\%)$ & $9(4.7)$ \\
\hline Norepinephrine, $n(\%)$ & $6(3.2)$ \\
\hline Dobutamine, $n(\%)$ & $8(4.2)$ \\
\hline Dopamine, $n(\%)$ & $1(0.5)$ \\
\hline \multicolumn{2}{|l|}{ Antithrombotic therapy at admission } \\
\hline Aspirin, $n(\%)$ & $190(100)$ \\
\hline Clopidogrel, $n(\%)$ & $57(30.0)$ \\
\hline Prasugrel, $n(\%)$ & $99(52.1)$ \\
\hline Ticagrelor, $n(\%)$ & $80(42.1)$ \\
\hline Fondaparinux, $n(\%)$ & $154(81.1)$ \\
\hline
\end{tabular}

Table 1 Demographic and clinical characteristics of the 190 study participants (Continued)

\begin{tabular}{ll}
\hline Unfractioned heparin, $n$ (\%) & $145(76.3)$ \\
Low molecular weight heparin, $n$ (\%) & $13(6.8)$ \\
Bivalirudin, $n$ (\%) & $2(1.1)$ \\
Abciximab, $n$ (\%) & $46(24.2)$ \\
Thrombolysis, $n$ (\%) & $2(1.1)$ \\
Beta-blocker therapy at admission, $n$ (\%) & $173(91.1)$ \\
Regular use of glucocorticoids, $n$ (\%) & $13(6.8)$ \\
Global Registry of Acute Coronary Events risk & $107(27)$ \\
score, M (SD) & \\
Acute distress during myocardial infarction, M (SD) & $6.23(1.40)$ \\
Sleep difficulties total, M (SD) & $1.53(1.29)$ \\
Difficulties initiating sleep, M (SD) & $1.24(1.62)$ \\
Nighttime awakenings, M (SD) & $2.53(2.10)$ \\
Difficulties maintaining sleep, M (SD) & $1.17(1.68)$ \\
Nonrestorative sleep, M (SD) & $1.17(1.67)$ \\
High risk of obstructive sleep apnea, $n$ (\%) & $77(40.5)$ \\
\hline
\end{tabular}

\section{Independent associations of sleep measures with} neuroendocrine hormones

Table 2 shows the fully adjusted multivariate model for the levels of neuroendocrine hormones. Compared to patients with low OSA risk, those with high OSA risk had significantly lower levels for all three hormones. Figure 1 shows absolute values of these group differences. More sleep difficulties (continuously scaled) were also significantly associated with lower NEPI levels (absolute values illustrated in Fig. 2 for poor vs. good sleepers). The relationship between sleep difficulties and NEPI was driven by difficulties initiating $\left(\mathrm{r}_{\mathrm{p}}=-0.15, p=0.049\right)$ and maintaining $\left(r_{p}=-0.20, p=0.008\right)$ sleep. Moreover, there was no significant interaction between OSA risk and sleep difficulties for stress hormones ( $p$-values $>0.36$ ).

\section{Independent associations of sleep measures with} coagulation factors

Table 3 shows the fully adjusted multivariate model for the three coagulation factors. More sleep difficulties were associated with greater fibrinogen levels (absolute values illustrated in Fig. 2 for poor vs. good sleepers), with difficulties initiating sleep driving this relationship $\left(r_{p}=0.25, p=0.001\right)$. Risk of OSA was not significantly associated with any coagulation factor. Likewise, there was no significant interaction between OSA risk and sleep difficulties for coagulation factors ( $p$-values $>0.35$ ).

\section{Role of stress hormones in the relation between sleep and coagulation factors}

The positive association of sleep difficulties with fibrinogen levels was virtually unchanged when EPI $\left(r_{p}=0.16\right.$, 
Table 2 Multivariate relations between sleep problems and neuroendocrine measures

\begin{tabular}{|c|c|c|c|c|c|c|}
\hline \multirow[t]{2}{*}{ Entered variables } & \multicolumn{2}{|l|}{ Epinephrine } & \multicolumn{2}{|c|}{ Norepinephrine } & \multicolumn{2}{|l|}{ Cortisol } \\
\hline & Partial corr. & P & Partial corr. & $P$ & Partial corr. & $P$ \\
\hline Age & -0.183 & 0.016 & 0.050 & 0.516 & -0.087 & 0.254 \\
\hline Male sex & -0.079 & 0.302 & 0.080 & 0.295 & -0.031 & 0.690 \\
\hline Education & -0.092 & 0.230 & -0.003 & 0.970 & -0.051 & 0.504 \\
\hline Charlson index & -0.240 & 0.001 & -0.126 & 0.098 & -0.111 & 0.147 \\
\hline Hypertension history & 0.159 & 0.037 & 0.088 & 0.247 & 0.139 & 0.068 \\
\hline High cholesterol history & 0.130 & 0.089 & -0.070 & 0.357 & -0.002 & 0.978 \\
\hline Depression history & 0.070 & 0.360 & 0.054 & 0.479 & 0.007 & 0.928 \\
\hline Body mass index & -0.053 & 0.489 & 0.178 & 0.019 & 0.101 & 0.187 \\
\hline Smoking & -0.047 & 0.541 & -0.021 & 0.786 & 0.030 & 0.693 \\
\hline Alcohol consumption & 0.026 & 0.731 & -0.126 & 0.098 & 0.025 & 0.747 \\
\hline Physical activity & -0.149 & 0.051 & -0.031 & 0.688 & 0.043 & 0.572 \\
\hline Previous Ml & 0.140 & 0.067 & 0.001 & 0.994 & 0.074 & 0.333 \\
\hline ST-elevation MI & 0.150 & 0.049 & 0.172 & 0.024 & 0.081 & 0.289 \\
\hline Stenotic coronary arteries & 0.074 & 0.334 & 0.116 & 0.130 & 0.232 & 0.002 \\
\hline GRACE risk score & 0.234 & 0.002 & 0.131 & 0.085 & 0.157 & 0.039 \\
\hline Acute distress & 0.210 & 0.006 & 0.170 & 0.025 & 0.029 & 0.706 \\
\hline Sleep difficulties & -0.054 & 0.482 & -0.172 & 0.024 & 0.094 & 0.217 \\
\hline High risk of OSA & -0.185 & 0.015 & -0.150 & 0.049 & -0.251 & 0.001 \\
\hline Model statistic & $\begin{array}{l}R^{2}=0.295 \\
F_{18,171}=3.97 \\
P<0.001\end{array}$ & & $\begin{array}{l}R^{2}=0.212 \\
F_{18,171}=2.55 \\
P=0.001\end{array}$ & & $\begin{array}{l}R^{2}=0.174 \\
F_{18,171}=2.00 \\
P=0.012\end{array}$ & \\
\hline
\end{tabular}

Linear regression model with all variables entered in one block. Significant correlation coefficients (Partial corr.) and $P$-values are given in bold GRACE Global Registry of Acute Cardiac Events, MI myocardial infarction, OSA obstructive sleep apnea

$p=0.043)$, NEPI $\left(\mathrm{r}_{\mathrm{p}}=0.16, p=0.031\right)$ and COR $\left(\mathrm{r}_{\mathrm{p}}=\right.$ $0.15, p=0.055)$ were separately added to the model in Table 3. Neuroendocrine hormones were not significantly predictive of fibrinogen $(p$-values $>0.06)$, D-dimer ( $p$-values $>0.07)$ and VWF ( $p$-values $>0.37)$ levels, independently of all other covariates. Also, OSA risk $(p$-values $>0.27)$ and sleep difficulties $(p$-values $>0.11)$ showed no significant interaction with neuroendocrine hormones for any coagulation factor.

\section{Discussion}

In patients admitted with acute $\mathrm{MI}$, we found a high risk of OSA to be associated with decreased levels of EPI, NEPI, and COR, compared to when OSA risk was low, independent of covariates. Sleep difficulties in terms of insomnia symptoms were also independently associated with low NEPI levels, although not with EPI and COR levels. Less evidence was found for an independent relationship between sleep problems and coagulation factors. Whereas insomnia symptoms were associated with higher levels of fibrinogen, although not with D-dimer and VWF, OSA risk did not show a significant association with any coagulation factor. The size of these associations was similar to that observed for several covariates with neuroendocrine and coagulation outcomes, including the prognostic GRACE score, implying not only statistical, but also clinical significance with small-to-moderate effects of sleep problems.

In our sample, $40.5 \%$ of patients had a high OSA risk, a prevalence similar to previous studies showing that close to a half of patients with ACS have moderate or severe OSA (apnea-hypopnea index $\geq 15$ ) [55]. Also comparable to a previous study showing that $37.3 \%$ of hospitalized patients with ACS have clinically relevant insomnia symptoms [56], 27.4\% of our patients were poor sleepers. In agreement with previous studies on CVD outcomes [34], we did not observe joint effects between OSA risk and insomnia symptoms for neuroendocrine hormones and coagulation factors. However, concurring with a high prevalence of OSA among insomnia patients and vice versa [32], we found a significant association between OSA risk and insomnia symptoms with frequent awakenings and non-restorative sleep driving this association. As we adjusted for an overlap between OSA risk and insomnia symptoms, we may interpret that in the setting of acute MI, neuroendocrine and coagulation abnormalities differ substantially between high OSA risk and insomnia symptoms 
Epinephrine plasma level

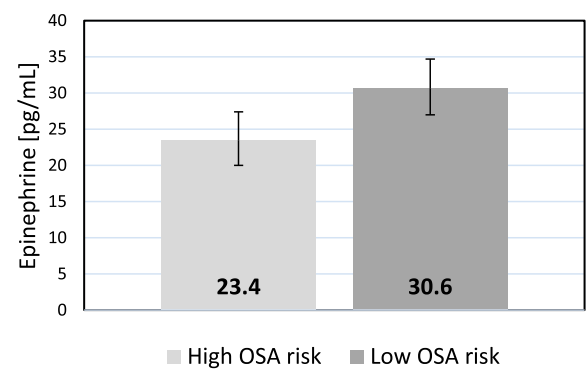

Norepinephrine plasma level

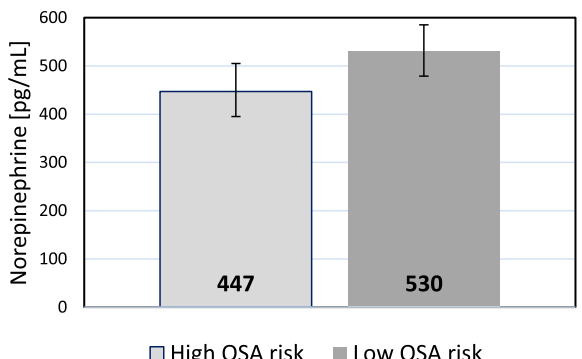

Cortisol serum level

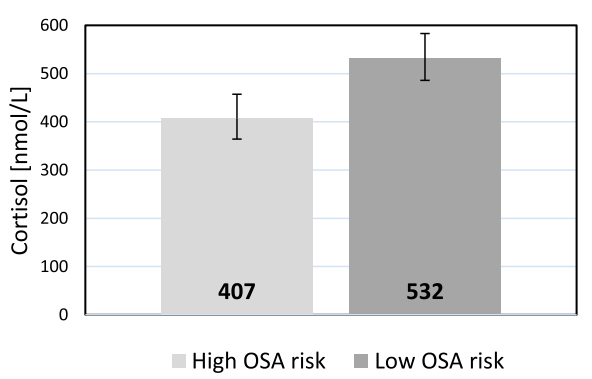

Fig. 1 Risk of obstructive sleep apnea and mean values of neuroendocrine measures. The bar graphs illustrate the significant differences in epinephrine $(p=0.015)$, norepinephrine $(p=0.049)$ and cortisol $(p=0.001)$ levels between subjects with high $(n=77)$ vs. low $(n=113)$ risk of obstructive sleep apnea (OSA), expressed as geometric mean values with 95\% confidence interval. Adjustments were made for age, sex, education, Charlson comorbidity index, positive history of hypertension/high cholesterol/depression, body mass index, smoking, alcohol consumption, physical activity, previous myocardial infarction (MI), ST-segment MI, number of stenotic coronary arteries, Global Registry of Acute Coronary Events risk score, acute distress, and sleep difficulties

and even between individual insomnia symptoms. We particularly found difficulties in initiating sleep to be significantly associated with NEPI and fibrinogen, and difficulties maintaining sleep also with NEPI, whereas waking up during the night and nonrestorative sleep showed no association with neuroendocrine hormones and coagulation factors whatsoever.

Increased fibrinogen levels in acute MI patients with more severe insomnia symptoms is compatible with a prothrombotic state, one mechanism that may possibly increase the risk of recurrent atherothrombotic events [21]. In addition to having rheological and hemostatic properties, fibrinogen is also an inflammatory molecule (i.e., an acute phase reactant), and inflammation and sleep disturbances are reciprocally linked with each other [57]. However, as we measured fibrinogen only once, but inquired about insomnia symptoms covering the preceding 4 weeks, we are unable to make any causal inferences. For instance, it is possible that insomnia symptoms were already associated with elevated fibrinogen before MI and/or even a contributing factor to MI onset, although exaggerated fibrinogen production during the acute phase could also have occurred in those with more severe insomnia symptoms. Clearly, our finding in fibrinogen needs replication, as we did not observe a relation between insomnia symptoms and the other two prothrombotic markers. Future studies should also include measures of impaired fibrinolysis, particularly plasminogen activator inhibitor-1 $[16,19]$.

Regarding neuroendocrine dysregulation, low endogenous COR levels in CHD patients with high OSA risk could result in less curtailing of vascular inflammation and atherosclerosis progression, respectively [58]. Indeed, low cortisol levels at admission were previously shown to predict early death in patients with acute MI [59]. Likewise, low catecholamine levels, found in those with high OSA risk and more sleep difficulties in our study, are implied in increased systemic inflammation [60]. Moreover, in OSA, due to the accompanying SNS hyperactivity, beta-2-adrenergic receptors are desensitized [61], a mechanism that may result in blunted beta-2-adrenergic receptor-mediated anti-inflammatory effects of catecholamines [60]. As coagulation activation relates to catecholamine and cortisol activity [30, 31], comparably low SNS and HPA activation in relation to sleep problems may help to explain why neuroendocrine dysregulation was no associated with coagulation factors in patients with high OSA risk and insomnia symptoms, respectively.

That patients with OSA might not mount an appropriate stress response to ACS could not only have 


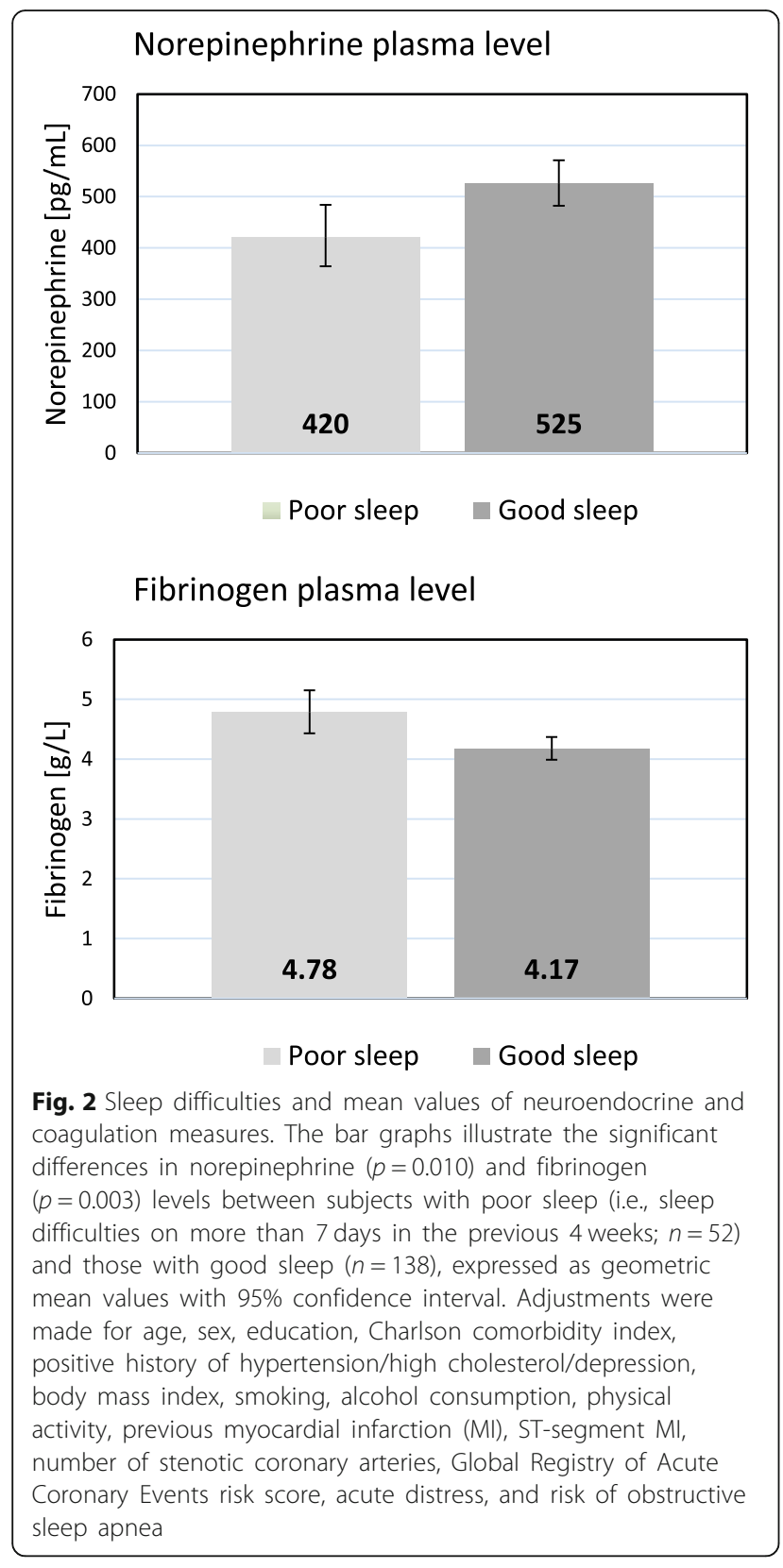

deleterious consequences for cardiac prognosis, as there is data from animal models to suggest that chronic intermittent hypoxia is a potentially adaptive stimulus for the myocardium in OSA [62]. One study in humans found more severe OSA to be associated with less cardiac injury during non-fatal ACS, proposing that ischemic preconditioning due to chronic intermittent hypoxia could be a possible explanation [63]. Although the involved physiological mechanisms are still elusive, there may be pro-angiogenic and anti-oxidant effects of intermittent hypoxia [62, 63]. For instance, as increased sympathetic tone and elevated catecholamine levels in OSA might be associated with increased reactive oxygen species production [64]. A lower sympathoadrenal response in the setting of ACS might result in less oxidative stress and myocardial damage, respectively.

Our study has several limitations worth mentioning. Most importantly, the lack of objective measures of sleep architecture, sleep duration and sleep apnea testing does not allow us to draw firm conclusions. To better differentiate between effects of OSA risk and insomnia symptoms, polysomnography or the additional inclusion of validated screening tools, such as the Berlin questionnaire and the Epworth Sleepiness Scale would have yielded data that are more reliable. We were particularly unable to tease apart influences of obstructive versus central sleep apnea that is prevalent in ACS. Moreover, objective short sleep duration is an important factor driving activation of the stress system and cardiometabolic morbidity in patients with insomnia [65]. However, it should also be mentioned that the acute cardiac situation influences sleep macro- and microarchitecture [66], which can complicate the diagnostic process. The STOP screening questionnaire is not very sensitive and specific for moderate-to-severe sleep-disordered breathing in CHD [42], but also in other disorders $[42,67]$ and in population-based studies [45]. A high sensitivity to detect more severe forms of OSA in patients with coronary artery bypass surgery was associated with low specificity [42], which increases the risk of false positives. The STOP questionnaire does not allow a firm assessment of OSA severity. Follow-up sleep studies would have been necessary for this purpose. It is not clear how the modified STOP questionnaire influenced its sensitivity and specificity. The omission of loudness of snoring may have overestimated the prevalence of patients classified with high OSA risk. Nonetheless, the majority of patients who reported a history of sleep apnea were in this group, suggesting our slightly modified STOP tool showed clinically useful sensitivity. Although time of blood collection did not significantly relate to coagulation and neuroendocrine measures, given their circadian variation, the difference in the time of assessment is a limitation. Although we applied an established method to replace missing data, the fact that $24 \%$ of patients missed catecholamine measures is a further limitation. We controlled for a range of important covariates, but residual confounding through for instance combined effects of emergency medications remains a possibility. This weakens the interpretation of the relationships which only emerged as significant in the covariate-adjusted analyses. In turn, non-significant results must be interpreted with caution, as we did not perform a power calculation for this ancillary sleep study. Body mass index, a driver of neuroendocrine and coagulation measures, was self-reported. Although we controlled for acute distress during MI, the 
Table 3 Multivariate relationship between sleep problems and coagulation factors

\begin{tabular}{|c|c|c|c|c|c|c|}
\hline \multirow[t]{2}{*}{ Entered variables } & \multicolumn{2}{|l|}{ Fibrinogen } & \multicolumn{2}{|l|}{ D-dimer } & \multicolumn{2}{|l|}{ WWF } \\
\hline & Partial corr. & $P$ & Partial corr. & $P$ & Partial corr. & $P$ \\
\hline$\overline{\text { Age }}$ & -0.059 & 0.439 & -0.125 & 0.102 & -0.006 & 0.942 \\
\hline Male sex & -0.073 & 0.343 & -0.083 & 0.278 & -0.114 & 0.137 \\
\hline Education & -0.190 & 0.012 & -0.096 & 0.208 & -0.018 & 0.810 \\
\hline Charlson index & 0.115 & 0.133 & 0.177 & 0.020 & 0.021 & 0.779 \\
\hline Hypertension history & 0.078 & 0.309 & 0.037 & 0.628 & -0.034 & 0.654 \\
\hline High cholesterol history & 0.041 & 0.591 & -0.063 & 0.409 & -0.049 & 0.522 \\
\hline Depression history & -0.178 & 0.019 & 0.037 & 0.627 & -0.060 & 0.432 \\
\hline Body mass index & 0.133 & 0.081 & -0.011 & 0.890 & 0.136 & 0.075 \\
\hline Smoking & 0.032 & 0.678 & -0.061 & 0.426 & 0.003 & 0.973 \\
\hline Alcohol consumption & -0.054 & 0.477 & 0.087 & 0.253 & 0.032 & 0.674 \\
\hline Physical activity & -0.090 & 0.238 & 0.069 & 0.367 & -0.012 & 0.877 \\
\hline Previous Ml & 0.047 & 0.538 & 0.155 & 0.042 & 0.082 & 0.286 \\
\hline ST-elevation MI & 0.158 & 0.038 & 0.019 & 0.800 & 0.010 & 0.898 \\
\hline Stenotic coronary arteries & 0.074 & 0.331 & -0.083 & 0.280 & 0.003 & 0.972 \\
\hline GRACE risk score & 0.102 & 0.180 & 0.279 & $<0.001$ & 0.276 & $<0.001$ \\
\hline Acute distress & 0.033 & 0.668 & 0.060 & 0.430 & 0.126 & 0.097 \\
\hline Sleep difficulties & 0.158 & 0.037 & 0.040 & 0.600 & 0.079 & 0.299 \\
\hline High risk of OSA & -0.038 & 0.618 & -0.082 & 0.283 & 0.017 & 0.828 \\
\hline Model statistic & $\begin{array}{l}R^{2}=0.216 \\
F_{18,171}=2.61 \\
P=0.001\end{array}$ & & $\begin{array}{l}R^{2}=0.245 \\
F_{18,171}=3.08 \\
P<0.001\end{array}$ & & $\begin{array}{l}R^{2}=0.236 \\
F_{18,171}=2.93 \\
P<0.001\end{array}$ & \\
\hline
\end{tabular}

Linear regression model with all variables entered in one block. Significant partial correlation coefficients (Partial corr.) and $P$-values are given in bold GRACE Global Registry of Acute Cardiac Events, MI myocardial infarction, OSA obstructive sleep apnea, VWF von Willebrand factor

specifics of our sample with substantially distressed patients need to be considered when generalizing the findings to other cohorts of ACS patients. Distressed patients may have over-reported sleep difficulties in their effort to make sense of the situation. However, about $70 \%$ of patients experience at least moderate distress and fear of dying during ACS [68]. As is common in ACS studies, less than 20\% of our patients were women, which precluded sex-stratified analyses, which might result in different findings for women and men $[10,11]$.

\section{Conclusions}

In patients admitted with acute MI, high OSA risk and insomnia symptoms might differently affect neuroendocrine and coagulation activity. While a high OSA risk was associated with neuroendocrine dysregulation, indicating SNS and HPA axis hypoactivity, insomnia symptoms, particularly problems initiating or maintaining sleep, showed a relation with reduced SNS activity and a prothrombotic state. Low cortisol and catecholamine levels might result in less curtailing of vascular inflammation, and elevated fibrinogen levels might evoke a prothrombotic state, with such pathophysiology helping to explain adverse outcome of MI patients with sleep problems. In turn, the finding of lower neuroendocrine hormones in patients with acute MI and a high OSA risk may also inform further studies to better understand potentially cardioprotective mechanisms of chronic intermittent hypoxia.

\section{Abbreviations}

ACS: Acute coronary syndrome; CHD: Coronary heart disease; COR: Cortisol; CVD: Cardiovascular disease; EPI: Epinephrine; GRACE: Global Registry of Acute Cardiac Events; HPA: Hypothalamic-pituitary adrenal; MI: Myocardial infarction; NEPI: Norepinephrine; OSA: Obstructive sleep apnea; SNS: Sympathetic nervous system; STEMI: ST-elevation myocardial infarction; WWF: Von Willebrand factor

\section{Acknowledgements}

Not applicable.

\section{Funding}

This study was financially supported by grant No. 140960 from the Swiss National Science Foundation to RVK (PI), JPS, US, HZ and JB. Additional support came from the Teaching and Research Directorate, Bern University Hospital, Switzerland.

\section{Availability of data and materials}

The dataset used and analyzed for the current study and to generate figures is available from the corresponding author on reasonable request. 


\section{Authors' contributions}

Rvk had full access to all data in the study and takes responsibility for the integrity of the data and the accuracy of the data analysis. RvK was responsible for statistical analysis and writing of the first draft of the manuscript. MP and REML were responsible for the acquisition of the data. RvK, MP, JPS, JB, HZ, US, and REML were responsible for the study concept and design, interpretation of data, critical revision of the manuscript for intellectual content, and approval of the manuscript for submission

\section{Ethics approval and consent to participate}

The study protocol was approved by the ethics committee of the State of Bern, Switzerland (KEK-Nr. 170/12). All patients were informed and gave signed consent.

\section{Consent for publication}

Not applicable.

\section{Competing interests}

The authors declare that they have no competing interests.

\section{Publisher's Note}

Springer Nature remains neutral with regard to jurisdictional claims in published maps and institutional affiliations.

\section{Author details}

'Department of Consultation-Liaison Psychiatry and Psychosomatic Medicine, University Hospital Zurich, Culmannstrasse 8, CH-8091 Zurich, Switzerland. ${ }^{2}$ Department of Cardiology, Clinic Barmelweid, Barmelweid, Switzerland. ${ }^{3}$ Complementary and Integrative Medicine, University of Zurich, Zurich, Switzerland. ${ }^{4}$ Department of Clinical Psychology and Psychotherapy, University of Bern, Bern, Switzerland. ${ }^{5}$ Medical Faculty, University of Zurich, Zurich, Switzerland. ${ }^{6}$ Department of Psychiatry, Clienia Schlössli AG, Oetwil am See, Switzerland.

Received: 26 July 2018 Accepted: 1 November 2018

Published online: 21 November 2018

\section{References}

1. Jordan AS, McSharry DG, Malhotra A. Adult obstructive sleep apnoea. Lancet. 2014;383(9918):736-47.

2. Sateia MJ. International classification of sleep disorders-third edition: highlights and modifications. Chest. 2014;146(5):1387-94.

3. Fu Y, Xia Y, Yi H, Xu H, Guan J, Yin S. Meta-analysis of all-cause and cardiovascular mortality in obstructive sleep apnea with or without continuous positive airway pressure treatment. Sleep Breath. 2017;21(1):181-9.

4. Wang X, Ouyang Y, Wang Z, Zhao G, Liu L, Bi Y. Obstructive sleep apnea and risk of cardiovascular disease and all-cause mortality: a meta-analysis of prospective cohort studies. Int J Cardiol. 2013;169(3):207-14.

5. He Q, Zhang P, Li G, Dai H, Shi J. The association between insomnia symptoms and risk of cardio-cerebral vascular events: a meta-analysis of prospective cohort studies. Eur J Prev Cardiol. 2017;24(10):1071-82.

6. Li M, Zhang XW, Hou WS, Tang ZY. Insomnia and risk of cardiovascular disease: a meta-analysis of cohort studies. Int J Cardiol. 2014;176(3):1044-7.

7. Sofi F, Cesari F, Casini A, Macchi C, Abbate R, Gensini GF. Insomnia and risk of cardiovascular disease: a meta-analysis. Eur J Prev Cardiol. 2014;21(1):57-64.

8. Xie W, Zheng F, Song X. Obstructive sleep apnea and serious adverse outcomes in patients with cardiovascular or cerebrovascular disease: a PRISMA-compliant systematic review and meta-analysis. Medicine (Baltimore). 2014;93(29):e336.

9. Zhao Y, Yu BY, Liu Y, Liu Y. Meta-analysis of the effect of obstructive sleep apnea on cardiovascular events after percutaneous coronary intervention. Am J Cardiol. 2017;120(6):1026-30.

10. Clark A, Lange T, Hallqvist J, Jennum P, Rod NH. Sleep impairment and prognosis of acute myocardial infarction: a prospective cohort study. Sleep. 2014;37(5):851-8.

11. Leineweber C, Kecklund G, Janszky I, Akerstedt T, Orth-Gomér K. Poor sleep increases the prospective risk for recurrent events in middle-aged women with coronary disease. The Stockholm female coronary risk study. J Psychosom Res. 2003;54(2):121-7.

12. Appels A, Kop W, Bär F, de Swart H, Mendes de Leon C. Vital exhaustion, extent of atherosclerosis, and the clinical course after successful percutaneous transluminal coronary angioplasty. Eur Heart J. 1995;16(12):1880-5.

13. Lévy P, Kohler M, McNicholas WT, Barbé F, McEvoy RD, Somers VK, Lavie L, Pépin L. Obstructive sleep apnoea syndrome. Nat Rev Dis Primers. 2015;1:15015.

14. Baltzis D, Bakker JP, Patel SR, Veves A. Obstructive sleep apnea and vascular diseases. Compr Physiol. 2016:6(3):1519-28.

15. Motivala SJ. Sleep and inflammation: psychoneuroimmunology in the context of cardiovascular disease. Ann Behav Med. 2011:42(2):141-52.

16. von Känel R, Loredo JS, Ancoli-Israel S, Mills PJ, Natarajan L, Dimsdale JE. Association between polysomnographic measures of disrupted sleep and prothrombotic factors. Chest. 2007;131(3):733-9.

17. Mausbach BT, Ancoli-Israel S, von Känel R, et al. Sleep disturbance, norepinephrine, and D-dimer are all related in elderly caregivers of people with Alzheimer disease. Sleep. 2006;29(10):1347-52.

18. von Känel R, Ancoli-Israel S, Dimsdale JE, et al. Sleep and biomarkers of atherosclerosis in elderly Alzheimer caregivers and controls. Gerontology. 2010:56(1):41-50.

19. Mehra R, Xu F, Babineau DC, Tracy RP, Jenny NS, Patel SR, Redline S. Sleepdisordered breathing and prothrombotic biomarkers: cross-sectional results of the Cleveland family study. Am J Respir Crit Care Med. 2010;182(6):826-33.

20. Shitrit D, Peled N, Shitrit AB, Meidan S, Bendayan D, Sahar G, Kramer MR. An association between oxygen desaturation and D-dimer in patients with obstructive sleep apnea syndrome. Thromb Haemost. 2005;94(3):544-7.

21. Toss H, Lindahl B, Siegbahn A, Wallentin L. Prognostic influence of increased fibrinogen and C-reactive protein levels in unstable coronary artery disease. FRISC study group. Fragmin during instability in coronary artery disease. Circulation. 1997;96(12):4204-10.

22. Lee KW, Lip GY, Tayebjee M, Foster W, Blann AD. Circulating endothelial cells, von Willebrand factor, interleukin-6, and prognosis in patients with acute coronary syndromes. Blood. 2005;105(2):526-32.

23. Oldgren J, Linder R, Grip L, Siegbahn A, Wallentin L. Coagulation activity and clinical outcome in unstable coronary artery disease. Arterioscler Thromb Vasc Biol. 2001;21(6):1059-64.

24. Brügger-Andersen T, Pönitz $\vee$, Staines $H$, Grundt $H$, Hetland $\varnothing$, Nilsen DW. The prognostic utility of D-dimer and fibrin monomer at long-term followup after hospitalization with coronary chest pain. Blood Coagul Fibrinolysis. 2008;19(7):701-7.

25. Dimsdale JE, Coy T, Ziegler MG, Ancoli-Israel S, Clausen J. The effect of sleep apnea on plasma and urinary catecholamines. Sleep. 1995;18(5):377-81.

26. Balbo M, Leproult $R$, Van Cauter $E$. Impact of sleep and its disturbances on hypothalamo-pituitary-adrenal axis activity. Int J Endocrinol. 2010;2010:759234.

27. Rodenbeck A, Huether G, Rüther E, Hajak G. Interactions between evening and nocturnal cortisol secretion and sleep parameters in patients with severe chronic primary insomnia. Neurosci Lett 2002;324(2):159-163.

28. Backhaus J, Junghanns K, Hohagen F. Sleep disturbances are correlated with decreased morning awakening salivary cortisol. Psychoneuroendocrinology. 2004:29(9):1184-91.

29. Kong AP, Chan NN, Chan JC. The role of adipocytokines and neurohormonal dysregulation in metabolic syndrome. Curr Diabetes Rev. 2006;2(4):397-407.

30. von Känel R, Dimsdale JE. Effects of sympathetic activation by adrenergic infusions on hemostasis in vivo. Eur J Haematol. 2000;65(6):357-69.

31. von Känel R, Kudielka BM, Abd-el-Razik A, Gander ML, Frey K, Fischer JE. Relationship between overnight neuroendocrine activity and morning haemostasis in working men. Clin Sci (Lond). 2004;107(1):89-95.

32. Luyster FS, Buysse DJ, Strollo PJ Jr. Comorbid insomnia and obstructive sleep apnea: challenges for clinical practice and research. J Clin Sleep Med. 2010;6(2):196-204

33. Gupta MA, Knapp K. Cardiovascular and psychiatric morbidity in obstructive sleep apnea (OSA) with insomnia (sleep apnea plus) versus obstructive sleep apnea without insomnia: a case-control study from a nationally representative US sample. PLoS One. 2014;9(3):e90021.

34. Luyster FS, Kip KE, Buysse DJ, Aiyer AN, Reis SE, Strollo PJ Jr. Traditional and nontraditional cardiovascular risk factors in comorbid insomnia and sleep apnea. Sleep. 2014;37(3):593-600

35. Zhang J, Ma RC, Kong AP, et al. Relationship of sleep quantity and quality with 24-hour urinary catecholamines and salivary awakening cortisol in healthy middle-aged adults. Sleep. 2011;34(2):225-33.

36. Jackowska M, Ronaldson A, Brown J, Steptoe A. Biological and psychological correlates of self-reported and objective sleep measures. J Psychosom Res. 2016:84:52-5. 
37. Meister R, Princip M, Schmid JP, Schnyder U, Barth J, Znoj H, Herbert C, von Känel R. Myocardial Infarction - Stress Prevention INTervention (MI-SPRINT) to reduce the incidence of posttraumatic stress after acute myocardial infarction through trauma-focused psychological counseling: study protocol for a randomized controlled trial. Trials. 2013;14:329.

38. Edmondson D, Richardson S, Falzon L, Davidson KW, Mills MA, Neria Y. Posttraumatic stress disorder prevalence and risk of recurrence in acute coronary syndrome patients: a meta-analytic review. PLoS One. 2012;7:e38915.

39. Edmondson D, von Känel R. Post-traumatic stress disorder and cardiovascular disease. Lancet Psychiatry. 2017;4(4):320-9.

40. Windecker S, Kolh P, Alfonso F, et al. 2014 ESC/EACTS Guidelines on myocardial revascularization: The Task Force on Myocardial Revascularization of the European Society of Cardiology (ESC) and the European Association for Cardio-Thoracic Surgery (EACTS)Developed with the special contribution of the European Association of Percutaneous Cardiovascular Interventions (EAPCI). Eur Heart J. 2014;35(37):2541-619.

41. Roffi M, Patrono C, Collet JP, et al. 2015 ESC Guidelines for the management of acute coronary syndromes in patients presenting without persistent STsegment elevation: Task Force for the Management of Acute Coronary Syndromes in Patients Presenting without Persistent ST-Segment Elevation of the European Society of Cardiology (ESC). Eur Heart J. 2016;37(3):267-315.

42. Chung F, Yegneswaran B, Liao $P$, et al. STOP questionnaire: a tool to screen patients for obstructive sleep apnea. Anesthesiology. 2008;108(5):812-21.

43. Nunes FS, Danzi-Soares NJ, Genta PR, Drager LF, Cesar LA, Lorenzi-Filho G. Critical evaluation of screening questionnaires for obstructive sleep apnea in patients undergoing coronary artery bypass grafting and abdominal surgery. Sleep Breath. 2015;19(1):115-22.

44. Haddad A. Validierung eines miniaturisierten Gerätes zum präoperativen Screening bei Patienten mit gesichertem oder mutmaßlichem obstruktiven Schlafapnoesyndrom und Validierung des sogenannten STOP-Fragebogens. Dissertation Medizinische Fakultät, Universität Duisburg-Essen, 2015.

45. Silva GE, Vana KD, Goodwin JL, Sherrill DL, Quan SF. Identification of patients with sleep disordered breathing: comparing the four-variable screening tool, STOP, STOP-Bang, and Epworth Sleepiness Scales. J Clin Sleep Med. 2011;7(5):467-72.

46. Jenkins CD, Stanton BA, Niemcryk SJ, Rose RM. A scale for the estimation of sleep problems in clinical research. J Clin Epidemiol. 1988;41(4):313-21.

47. Ehrenreich $\mathrm{H}$, Schuck J, Stender N, et al. Endocrine and hemodynamic effects of stress versus systemic CRF in alcoholics during early and medium term abstinence. Alcohol Clin Exp Res. 1997;21(7):1285-93.

48. Coen Herak D, Milos M, Zadro R. Evaluation of the Innovance D-DIMER analytical performance. Clin Chem Lab Med. 2009:47(8):945-51.

49. Bopp M, Minder CE. Swiss National Cohort. Mortality by education in German speaking Switzerland, 1990-1997: results from the Swiss National Cohort. Int J Epidemiol. 2003:32(3):346-54.

50. Charlson ME, Pompei $P$, Ales KL, CR MK. A new method of classifying prognostic comorbidity in longitudinal studies: development and validation. J Chronic Dis. 1987;40(5):373-83.

51. Fox KA, Dabbous OH, Goldberg RJ, et al. Prediction of risk of death and myocardial infarction in the six months after presentation with acute coronary syndrome: prospective multinational observational study (GRACE). BMJ. 2006;333(7578):1091.

52. von Känel R, Hari R, Schmid JP, Saner H, Begré S. Distress related to myocardial infarction and cardiovascular outcome: a retrospective observational study. BMC Psychiatry. 2011;11:98.

53. Perneger TV. What's wrong with Bonferroni adjustments? BMJ. 1998;316:1236-8.

54. Cohen J. Statistical power for the behavioral sciences, ed 3. New York: Academic Press; 1988.

55. Le Grande MR, Neubeck L, Murphy BM, Mclvor D, Lynch D, McLean H, Jackson AC. Screening for obstructive sleep apnoea in cardiac rehabilitation: a position statement from the Australian Centre for Heart Health and the Australian cardiovascular health and rehabilitation association. Eur J Prev Cardiol. 2016;23(14):1466-75.

56. Coryell VT, Ziegelstein RC, Hirt K, Quain A, Marine JE, Smith MT. Clinical correlates of insomnia in patients with acute coronary syndrome. Int Heart J. 2013;54(5):258-65.

57. Irwin MR, Opp MR. Sleep Health: Reciprocal regulation of sleep and innate immunity. Neuropsychopharmacology. 2017;42(1):129-55.

58. Waller C, Bauersachs J, Hoppmann U, et al. Blunted cortisol stress response and depression-induced hypocortisolism is related to inflammation in patients with CAD. J Am Coll Cardiol. 2016;67(9):1124-6.
59. Reynolds RM, Walker BR, Haw S, et al. Low serum cortisol predicts early death after acute myocardial infarction. Crit Care Med. 2010:38(3):973-5.

60. Elenkov IJ. Neurohormonal-cytokine interactions: implications for inflammation, common human diseases and well-being. Neurochem Int 2008;52(1-2):40-51.

61. Mills PJ, Dimsdale JE, Coy TV, Ancoli-lsrael S, Clausen JL, Nelesen RA. Beta 2adrenergic receptor characteristics in sleep apnea patients. Sleep. 1995;18(1):39-42.

62. Yin X, Zheng Y, Liu Q, Cai J, Cai L. Cardiac response to chronic intermittent hypoxia with a transition from adaptation to maladaptation: the role of hydrogen peroxide. Oxidative Med Cell Longev. 2012;2012:569520.

63. Shah N, Redline S, Yaggi HK, et al. Obstructive sleep apnea and acute myocardial infarction severity: ischemic preconditioning? Sleep Breath. 2013, 17(2):819-26.

64. Khayat R, Patt B, Hayes D Jr. Obstructive sleep apnea: the new cardiovascular disease. Part l: obstructive sleep apnea and the pathogenesis of vascular disease. Heart Fail Rev. 2009;14(3):143-53.

65. Vgontzas AN, Fernandez-Mendoza J, Liao D, Bixler EO. Insomnia with objective short sleep duration: the most biologically severe phenotype of the disorder. Sleep Med Rev. 2013;17(4):241-54.

66. Schiza SE, Simantirakis E, Bouloukaki I, et al. Sleep patterns in patients with acute coronary syndromes. Sleep Med. 2010;11(2):149-53.

67. Westlake K, Plihalova A, Pretl M, Lattova Z, Polak J. Screening for obstructive sleep apnea syndrome in patients with type 2 diabetes mellitus: a prospective study on sensitivity of Berlin and STOP-bang questionnaires. Sleep Med. 2016;26:71-6.

68. Whitehead DL, Strike P, Perkins-Porras L, Steptoe A. Frequency of distress and fear of dying during acute coronary syndromes and consequences for adaptation. Am J Cardiol. 2005;96(11):1512-6.

\section{Ready to submit your research? Choose BMC and benefit from:}

- fast, convenient online submission

- thorough peer review by experienced researchers in your field

- rapid publication on acceptance

- support for research data, including large and complex data types

- gold Open Access which fosters wider collaboration and increased citations

- maximum visibility for your research: over $100 \mathrm{M}$ website views per year

At BMC, research is always in progress.

Learn more biomedcentral.com/submissions 